\title{
Mastering alien etiquette
}

After Contact: The Human Response to Extraterrestrial Life by Albert A. Harrison

Plenum: 1997. Pp. 363. \$28.95, £17.55

\section{PhilMorrison}

We will know little about extraterrestrials even after prolonged contact. But Albert A. Harrison, professor of psychology at the University of California, Davis, offers us reason enough to justify forecasting what they might be like.

The most striking impression made by this beautifully argued and warm-hearted book is one of subtle unity among humans, extended by probable inference to the 'others'. We are not at all the same, but disparate minds can enrich each other.

The book closes with a terse surprise. The two cultures once perceived by C. P. Snow are now in some degree testable. The Meyer-Briggs type indicator offers an objective measure of Jungian psychological types, and classifies 55 per cent of psychiatrists and social scientists as 'feelers', whereas more than 60 per cent of engineers and scientists are scored as 'thinkers'. Feelers "make decisions on broad personal and social values"; the rest "prefer impersonal, logical bases for decision".

True to type, this physicist reader is a little sceptical. It is far from easy to learn how people make decisions. Perhaps what counts is some weighted sum of life experiences. Harrison's book shows that he shares important traits with feelers, as he focuses easily on emotional responses and will never carelessly hurt another's feelings.

Yet he is attuned to a broader material consistency beyond the richness of social experience. Once he was washed in the sudden light of a fireball as bright as the Moon, another time puzzled by a restored fighter plane from the Second World War that he had mistaken for a model plane flying nearby, and he has long been charmed by the narrow intensities of amateur radio. He cites and follows the rationally structured theory of living systems put forward by James Grier Miller which rests explicitly on continuity among the sciences. His fascinating volume links him to what I conjecture to be the majority among Nature readers.

A dozen chapters, each of about 30 pages and almost without visuals, weigh first the physical bases of the search for interstellar signals, then speculations on the likely nature of extraterrestrial society, and finally the human responses we may expect. Harrison offers a well-documented and expressive summary of this magpie literature, avoiding both bland citation and simple dismissal. A too-small sample follows.

\section{Destination space}

This parody of the space race appeared in the Washington Post in 1961, and is reproduced in ...The Heavens and the Earth, Walter A. McDougall's magisterial political history of the US, European and Soviet space programmes, now reissued in paperback (Johns Hopkins University Press, $\$ 19.95, \mathfrak{E} 15.95)$. Some ten years on, the author reflects that he should have been gloomier, and that from today's vantage point the space age may well be defined as an era of hubris. To restart it, he says, we must "discover some new principle that makes spaceflight genuinely cheap, safe and routine... however it is achieved, a replacement for the old-fashioned rocket is needed to restore the political will and public support for a second era of 'swashbuckling' in space."

In October 1996 the World-Wide Web contained 20,000 references to unidentified flying objects (UFOs). One form of evidence of extraterrestrial life would be new and verifiable knowledge, so far missing from that haystack of chatter and promises. In Harrison's opinion, the whole UFO effort - perhaps millions of hours - has not added much support to tales from the 1940s. Repeated radio contact is verifiable, and can be shared widely if it comes, to puncture the bubble of false alarms.

Will war follow contact? Some have argued that only one predator society among very many other, benign societies would guarantee disaster. But would one evil empire induce no opposition? A few societies devoted to peace might well prevail. If information is the main basis for exchange across the star-set kiloparsecs, then "hurl threats and I may turn my radio off. Present me with a vision that I like, and I might stay tuned."

Contact will have short-term effects that depend greatly on detailed circumstances. Of the public events that led many to accept the existence of extraterrestrials, only Orson Welles's vivid radio play on Hallowe'en in 1938, as the Second World War threatened, led to a panicky reaction. It aired news of combat against armed Martians in New Jersey. Remakes saw that reaction repeated on several occasions in other countries, most recently in 1988.
"Fill 'Er Up-r'm in a Race"

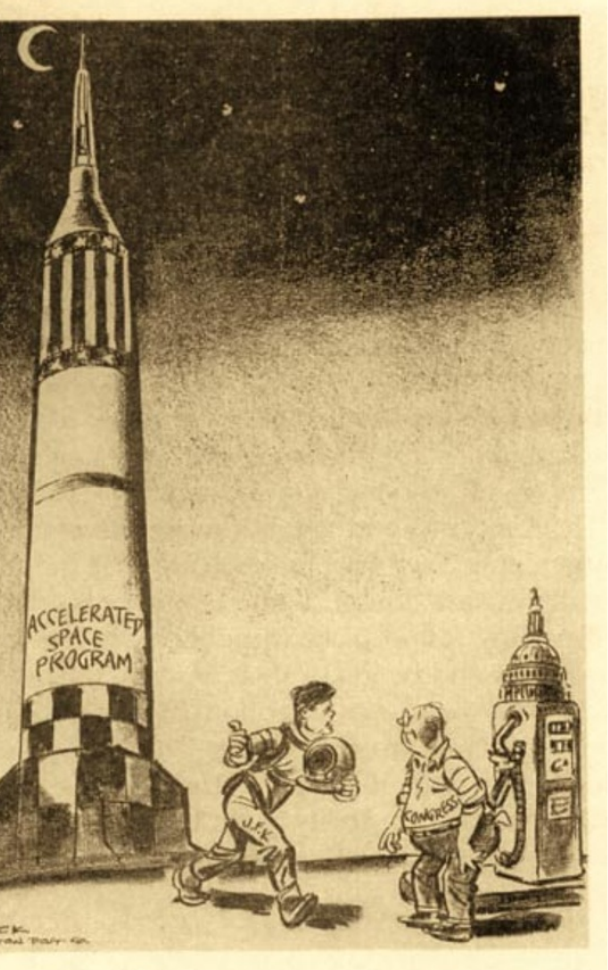

But reports in 1835 of carefree lunar 'bat men' acted mainly to sell copies of the hoaxing New York Sun. A network of canals on Mars became famous in the 1890s, but gondoliers there were none. Quasars and pulsars - even if controlled by little green men were mainly science news. Not even the current epidemic of private abductions by UFOs has stirred urgent outcry in the United States.

The most likely form of contact ahead, distant signals from beings unseen and very far away, suggests a brief media circus. The find may come at last to complete our postCopernican mindset, but only after lengthy decipherment and study.

Harrison ends with some moderate conclusions: if others exist, we will eventually uncover indisputable evidence. They will be recognizable social organisms, and to some extent we will understand them. Our first verifiable contact is more likely to be well handled than it is to incite panic. Their new ideas will not magically cure our ills, but are more likely to help us than to hurt.

So far, I have given only a physicist's paraphrase. But here are his own closing lines: "When we come right down to it, we should place our bets on the side of the optimists.... Let's stay tuned and find out!"

Phil Morrison is at Room 6-205, Massachusetts Institute of Technology, 77 Massachusetts Avenue, Cambridge, Massachusetts 02139, USA. 